\title{
Narrow Column of Contrast or Air Between Tongue Base and Posterior Pharyngeal Wall During Swallowing
}

National Cancer Institute

\section{Source}

National Cancer Institute. Narrow Column of Contrast or Air Between Tongue Base and Posterior Pharyngeal Wall During Swallowing. NCI Thesaurus. Code C127327.

A finding of a narrow column of contrast or air between tongue base and posterior pharyngeal wall during swallowing. 